\title{
Ferromagnetic Chain Based on Verdazyl-Nitroxide Diradical
}

Evgeny V. Tretyakov, ${ }^{[\mathrm{a}, \mathrm{b}]}$ Svetlana I. Zhivetyeva, ${ }^{[\mathrm{a}]}$ Pavel V. Petunin, ${ }^{[\mathrm{c}, \mathrm{d}]}$ Dmitry E. Gorbunov, ${ }^{[\mathrm{b}, \mathrm{e}]}$ Nina P. Gritsan, ${ }^{[\mathrm{b}, \mathrm{e}]}$ Irina Yu. Bagryanskaya,${ }^{[\mathrm{a}, \mathrm{b}]}$ Artem S. Bogomyakov,${ }^{[\mathrm{f}]}$ Pavel S. Postnikov, ${ }^{[\mathrm{d}, \mathrm{g}]}$ Maxim S. Kazantsev, ${ }^{[a, b]}$ Marina E. Trusova, ${ }^{[\mathrm{d}]}$ Inna K. Shundrina, ${ }^{[a, b]}$ Elena V. Zaytseva, ${ }^{[a, b]}$ Dmitriy A. Parkhomenko, ${ }^{[a, b]}$ Elena G. Bagryanskaya, ${ }^{[a, b]}$ Victor I. Ovcharenko* ${ }^{[\mathrm{f}]}$

[a] Prof. Dr. E.V. Tretyakov, Dr. S.I. Zhivetyeva, Prof. Dr. I.Yu. Bagryanskaya, Dr. M.S. Kazantsev, Dr. I.K. Shundrina, Dr. E.V. Zaytseva, Dr. D.A. Parkhomenko, Prof. Dr. E.G. Bagryanskaya N.N. Vorozhtsov Novosibirsk Institute of Organic Chemistry

9 Ac. Lavrentiev Avenue, Novosibirsk 630090, Russia

E-mail: tretyakov@nioch.nsc.ru

[b] Prof. Dr. E.V. Tretyakov, Dr. D.E. Gorbunov, Prof. Dr. N.P. Gritsan, Prof. Dr. I.Yu. Bagryanskaya, Dr. M.S. Kazantsev, Dr. I.K. Shundrina, Dr. E.V. Zaytseva, Dr. D.A. Parkhomenko, Prof. Dr. E.G. Bagryanskaya Novosibirsk State University 2 Pirogova Str., Novosibirsk 630090, Russia

[c] Dr. P.V. Petunin

Department of Chemistry

Siberian State Medical University

2 Moskovskiy Trakt, Tomsk 634050, Russia

[d] Dr. P.V. Petunin, Dr. P.S. Postnikov, Prof. Dr. M.E. Trusova

Research School of Chemistry \& Applied Biomedical Sciences

Tomsk Polytechnic University

30 Lenin Avenue, Tomsk 634050, Russia

E-mail: postnikov@tpu.ru

[e] Dr. D.E. Gorbunov, Prof. Dr. N.P. Gritsan

Voevodsky Institute of Chemical Kinetics and Combustions

3 Institutskaya Str., Novosibirsk 630090, Russia

[f] Dr. A.S. Bogomyakov, Prof. Dr. Victor I. Ovcharenko

International Tomography Center

3a Institutskaya Str., Novosibirsk 630090, Russia

E-mail: Victor.Ovcharenko@tomo.nsc.ru

[g] Dr. P.S. Postnikov

University of Chemistry and Technology

3 Technicka, Prague16628, Czech Republic 
Abstract: Verdazyl-nitroxide diradicals were synthesized using the palladium-catalyzed crosscoupling reaction of the corresponding iodoverdazyls with a nitronyl nitroxide-2-ide gold(I) complex with high yields (up to 82\%). The synthesized diradicals were found to be highly thermally stable and have a singlet $\left(\Delta E_{\mathrm{ST}} \approx-64 \mathrm{~cm}^{-1}\right)$ or triplet ground state $\left(\Delta E_{\mathrm{ST}} \geq 25\right.$ and $100 \mathrm{~cm}^{-1}$ ), depending on which canonical hydrocarbon diradical type they belong to. Upon crystallization, triplet diradicals form unique one-dimensional (1D) spin $S=1$ chains of organic diradicals with intrachain ferromagnetic coupling of $J^{\prime} / k_{\mathrm{B}}$ from 3 to $6 \mathrm{~K}$.

Diradicals are considered as promising building blocks for multifunctional molecule-based magnetic materials and the design of spintronic devices. ${ }^{[1]}$ The synthesis of stable high-spin organic molecules with a substantial energy gap between the high-spin ground state and the first excited low-spin state is of particular interest. The literature describes several such long-lived open-shell molecules which were isolated individually and fully characterized. ${ }^{[2]}$ In Figure 1 are shown real paramagnetic molecules combined with their triplet canonical diradical prototypes: ${ }^{[3]}$ 2-methylprop-1-en-1,2-diyl (MPD) and benzene-1,3-dimethyl (BDM; Figure 1). Recently, an important concept concerning close relation between quantum interference in molecular conductance and diradical existence was reported. ${ }^{[4]}$ This conceptual bridge between molecular conductance and diradical existence is extremely useful for the rational design of diradicals as working elements for quantum informatics. ${ }^{[5]}$ However, at the moment it is quite sophisticated to predict the parking motif in diradical solids that could serve potentially as an excellent systems for studies of low-dimensional magnetism. Not long ago, thermally stable diradical with a large singlet-triplet energy gap that consists of antiferromagnetic Heisenberg chains at low temperature and possesses record intrachain antiferromagnetic coupling $\left(J^{\prime} / k=-14 \mathrm{~K}\right)$ was describe. ${ }^{[2 \mathrm{a}]} \mathrm{We}$ succeeded in synthesizing verdazyl-nitroxide diradicals $(2,3)$ which, to the best of our knowledge, are the first examples of a 1D ferromagnetic $S=1$ chains. In this paper, we present the preparation and characterization of synthesized compounds.

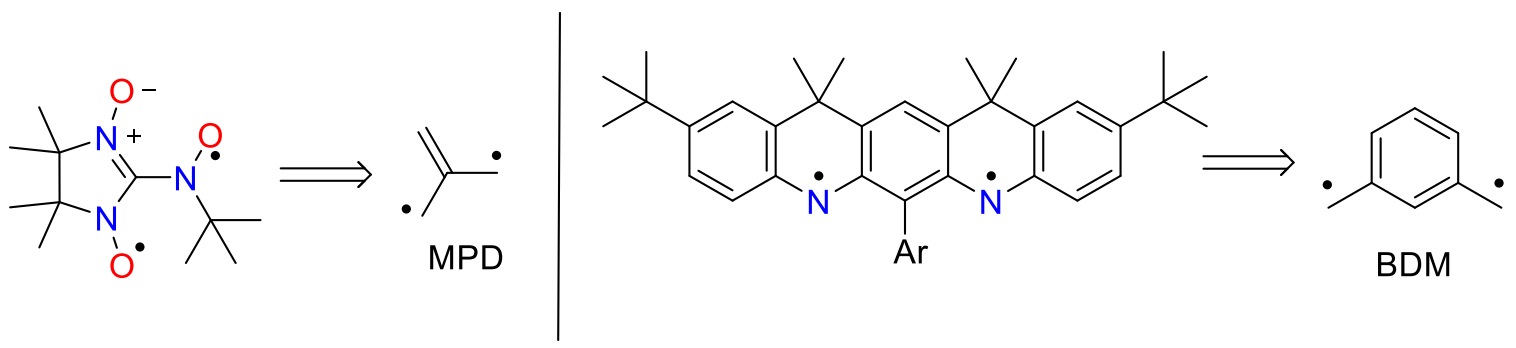

Figure 1. Examples of stable triplet diradicals and their prototypes. 
Depending on interconnection of building blocks, diradicals 1-3 are related to various hydrocarbon prototypes (Figure 2). According to prototype structures, we expected an intramolecular ferromagnetic exchange interaction in 2-4 and a weak antiferromagnetic one in $\mathbf{1}$. Indeed, density functional theory (DFT) calculations predicted a triplet ground state for $\mathbf{2}, \mathbf{3}$ and $\mathbf{4}$ with $\Delta E_{\mathrm{ST}}=1.36,0.37$, and $0.91 \mathrm{kcal} / \mathrm{mol}$, respectively, and a singlet ground state with $\Delta E_{\mathrm{ST}}=-$ $0.69 \mathrm{kcal} / \mathrm{mol}$ for diradical 1 (Supporting Information Section S1).
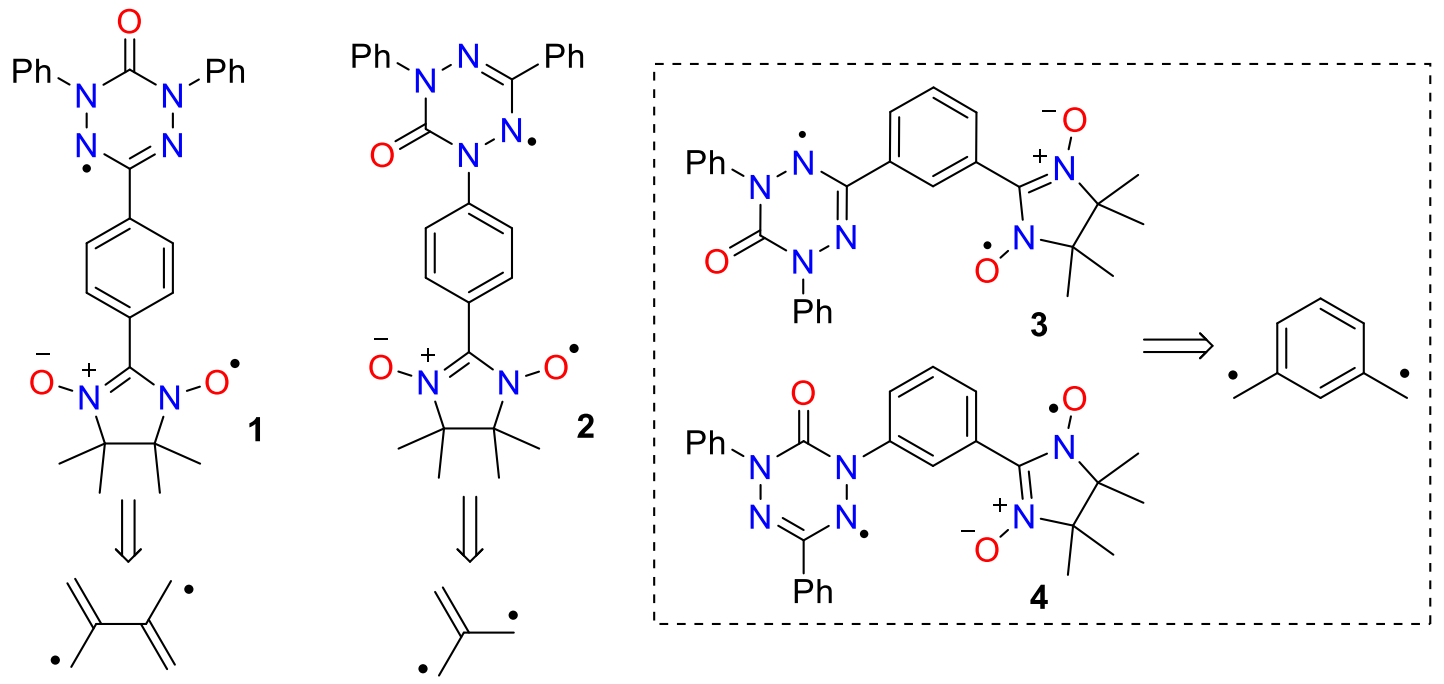

Figure 2. Proposed (nitronyl nitroxide)-oxoverdazyl diradicals 1-4 and their prototypes (stepby-step retro-transformation is shown in SI Section S1).

We succeeded in synthesizing 1-3 but not $\mathbf{4}$ (due to difficulties in preparation of the corresponding iodoverdazyl). To prepare diradicals 1-3, we applied a palladium-catalyzed cross-coupling reaction of iodoverdazyls 5-7 $\mathbf{7}^{[6]}$ with a nitronyl nitroxide-2-ide gold(I) complex ${ }^{[7]}$ (Scheme 1). Experimental procedures are given in Supporting Information Section S2. Desired diradicals 1-3 were stable at ambient conditions that allowed one to isolate them in pure form and completely characterize.
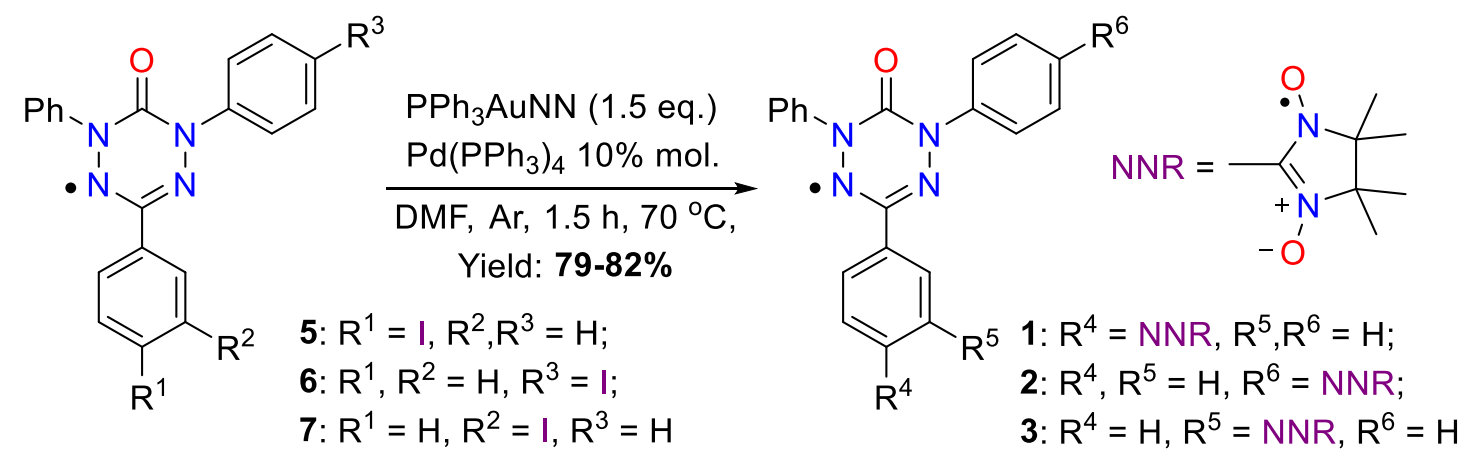

Scheme 1. Synthesis of diradicals 1-3. 
Molecular and crystal structures of 1-3 were solved by single-crystal X-ray diffraction (XRD) analysis. DFT calculations using XRD geometry revealed that the spin density in all diradicals is mainly localized on the nitrogen and oxygen atoms of two radical moieties, as shown, for example, for diradical 2 (Figure 3). Spin-distribution and SOMO localization for diradicals $\mathbf{1}-\mathbf{3}$ are reported in Supporting Information Section S1.
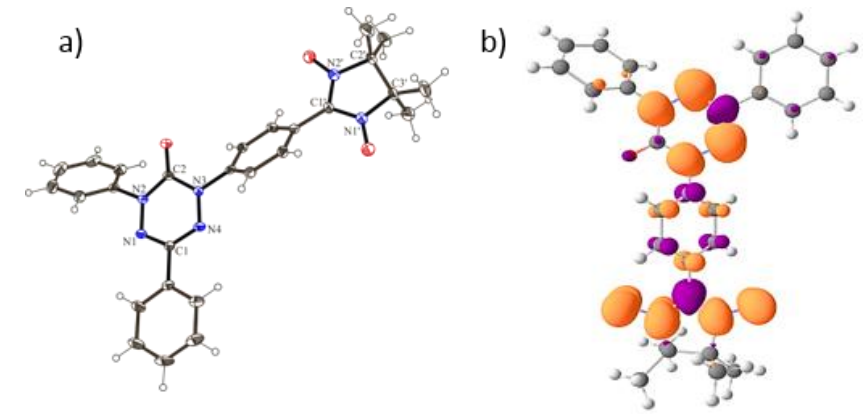

Figure 1. a) Single-crystal X-ray geometry of $\mathbf{2}$ with thermal ellipsoids shown at the $50 \%$ probability level. b) The DFT isosurface of electron spin density is indicated in orange (+) and pink (-) on top of the molecular geometry.

EPR spectra of diradicals 1-3 were recorded in toluene solutions at $295 \mathrm{~K}$ and in frozen matrices at $130 \mathrm{~K}$; Figure 4 shows the EPR spectra of triplet diradical 2. Room temperature spectra (Figure 4a and Supporting Information Section S3) can be reproduced quite well by means of spinHamiltonian parameters of the corresponding monoradical precursors and by taking into account that the intramolecular exchange interactions are much greater than hyperfine terms $\left(J>A_{N}\right)$. Low-temperature spectra (Figure 4b and SI Section S3) can be well simulated as those of triplet species with axial symmetry $(\mathrm{E}=0)$ taking into account zero-field splitting and unresolved hyperfine splitting. The diradical nature of 1-3 was additionally confirmed by detection of weak forbidden $\Delta \mathrm{Ms}=2$ transition; intensities of the latter are proportional to the zero-field splitting parameters differing significantly among the diradicals. 


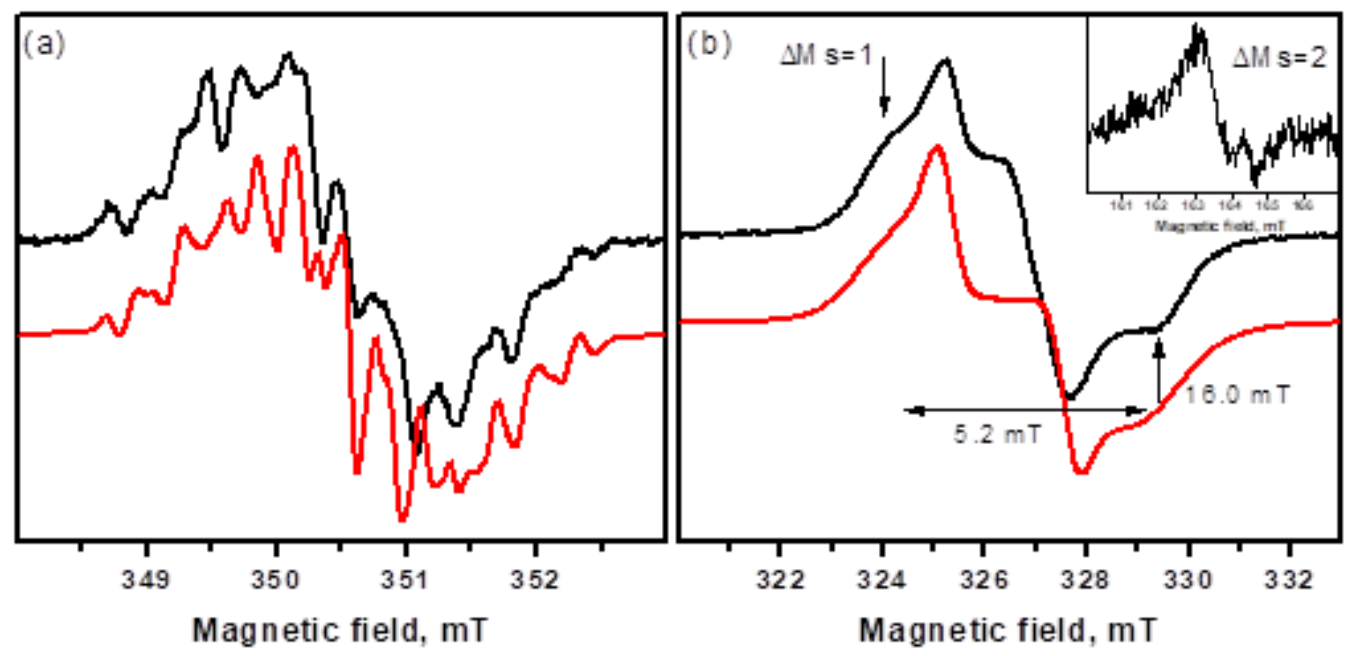

Figure 4. EPR spectra of 2 recorded from diluted and oxygen-free toluene solutions at $295 \mathrm{~K}$ (a) and at $130 \mathrm{~K}$ (b). Black curves - experimental spectra, red curves - their simul ation. Simulation parameters are given in SI Section S3.

Since $\mathbf{2}$ has the highest singlet-triplet energy gap among all the prepared diradicals $\mathbf{1}-\mathbf{3}$, we tested its stability using TGA-DSC tandem analysis in He atmosphere. Decomposition of diradical 2 was observed at $\sim 195.5{ }^{\circ} \mathrm{C}$ (the first TGA weight loss) meaning stability up to $195{ }^{\circ} \mathrm{C}$ in an inert atmosphere (Figure 5). According to EPR and infrared spectroscopy in the He atmosphere, diradical 2 is stable, at least up to $110^{\circ} \mathrm{C}$, as confirmed by comparing the corresponding spectra before and after heating (Supporting Information Section S4).

TG $/ \%$

$\mathrm{DSC} /(\mathrm{mW} / \mathrm{mg})$

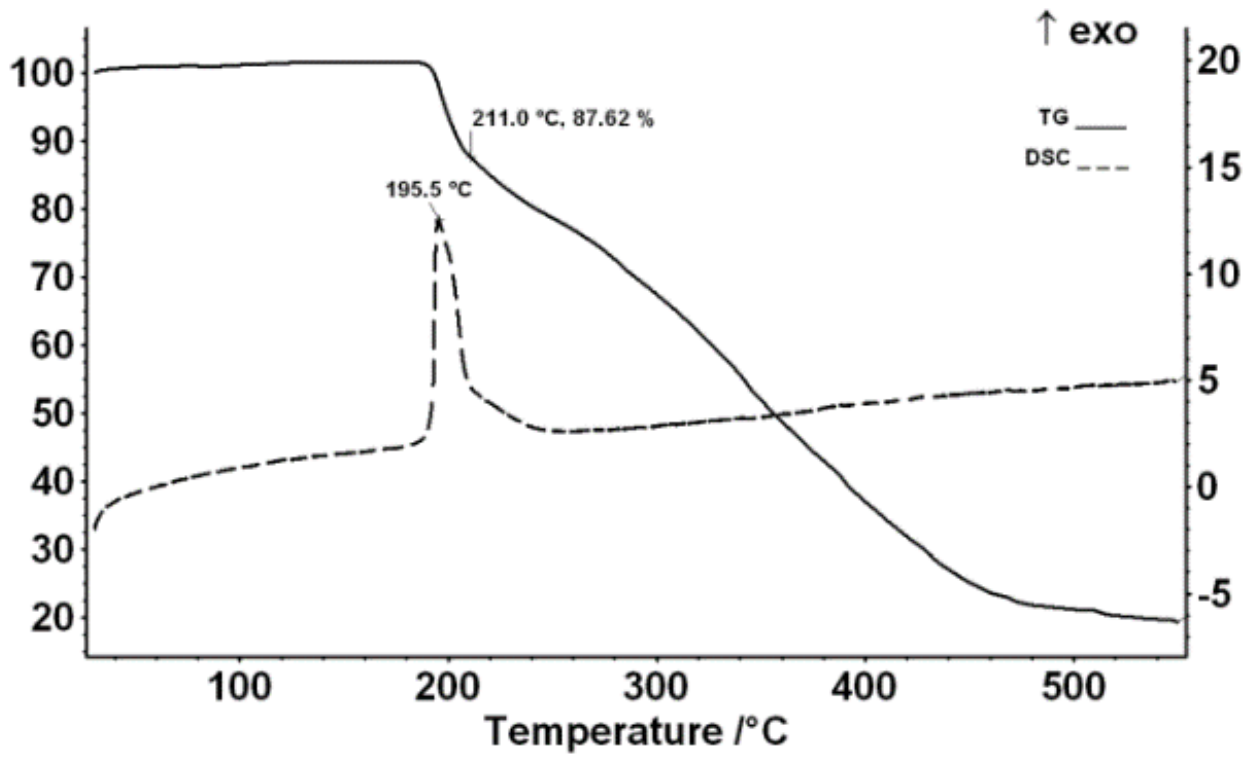

Figure 5. Thermogravimetric analysis (TGA) and differential scanning calorimetry (DSC) of diradical 2 under $\mathrm{He}$; heating rate $=10^{\circ} \mathrm{C} \cdot \mathrm{min}^{-1}$. 
Electrochemical properties of diradicals $\mathbf{1}-\mathbf{3}$ were studied by cyclic voltammetry in $\mathrm{CH}_{2} \mathrm{Cl}_{2}$ solutions (Figure 6). These mixed diradicals manifest similar redox behavior with two close reversible oxidation waves with almost identical $\mathrm{E}^{1 / 2}=0.4-0.6 \mathrm{~V}$ and two reduction waves corresponding to the verdazyl (reversible, at approximately $-1.0 \mathrm{~V}$ ) and nitronyl nitroxide (irreversible at approximately $-1.5 \mathrm{~V}$ ) moieties. The corresponding half-wave potentials are summarized in Table 1; the results indicate a negligible effect of the linker between the radical fragments on the electrochemical properties of the prepared compounds.

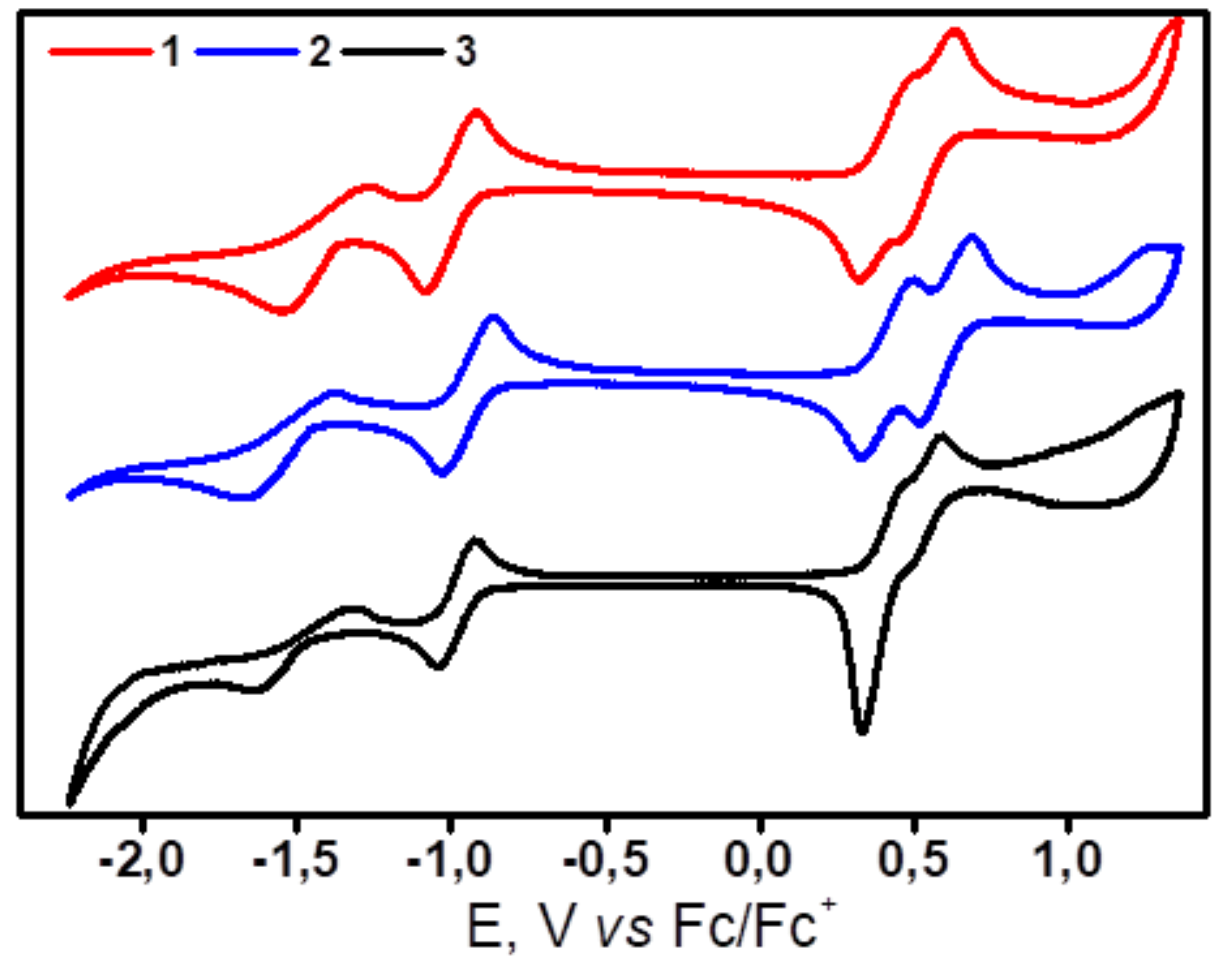

Figure 6. Cyclic voltammograms of diradicals $\mathbf{1}-\mathbf{3}$ in $\mathrm{CH}_{2} \mathrm{Cl}_{2}$ solutions $\left(0.1 \mathrm{~V} / \mathrm{s}, 0.1 \mathrm{M} \mathrm{Bu}_{4} \mathrm{NPF}_{6}\right.$ electrolyte).

Table 1. Oxidation and reduction potentials $\left(v s \mathrm{Fc} / \mathrm{Fc}^{+}\right)$of diradicals $\mathbf{1}-\mathbf{3}$ in comparison with initial compounds.

\begin{tabular}{lllll}
\hline Compound & $\mathrm{E}_{\text {Red } 1}^{1 / 2}, \mathrm{~V}$ & $\mathrm{E}_{\mathrm{Red} 2}^{1 / 2}, \mathrm{~V}$ & $\mathrm{E}_{\text {Ox } 1}^{1 / 2}, \mathrm{~V}$ & $\mathrm{E}_{\text {Ox2 }}^{1 / 2}, \mathrm{~V}$ \\
\hline $\mathbf{1}$ & -1.43 & -1.00 & 0.40 & 0.55 \\
$\mathbf{2}$ & -1.52 & -0.94 & 0.40 & 0.60 \\
$\mathbf{3}$ & -1.52 & -0.98 & 0.39 & 0.55 \\
Oxoverdazyls[a] & - & -1.00 & - & 0.50 \\
Nitronyl nitroxides [a] & -1.40 & - & 0.40 & - \\
\hline
\end{tabular}

[a] Typical values are given 
To predict magnetic motifs for crystalline samples of $\mathbf{1}-\mathbf{3}$, we performed broken-symmetry DFT (BS-DFT) and accurate CASSCF/NEVPT2 calculations of intra- and intermolecular exchange interactions using XRD data (Table 2 and SI Section S1). According to the calculations, 2 and 3 form infinite chains of ferromagnetically coupled diradicals. In the case of $\mathbf{3}$, the verdazyl moieties are coupled with both the verdazyl and nitronyl nitroxide parts $\left(J_{1} \approx J_{1^{\prime}} \approx 10 \mathrm{~cm}^{-1}\right)$ of neighboring diradicals within a chain (Figure S1.5). Only verdazyl fragments are noticeably coupled in chains of 2 (Figure 7). In crystals of both 2 and 3, apparent antiferromagnetic interactions between nitronyl nitroxide moieties of the neighboring chains were predicted (Figure 7 and SI Section S1).

Table 2. Parameters of the intramolecular $\left(J_{\text {dir }}\right)$ and intra- $\left(J_{1}\right)$ and inter-chain $\left(J_{2}\right)$ exchange interactions $\left(\widehat{H}=-2 J \widehat{\overrightarrow{S_{1}} \widehat{\overrightarrow{S_{2}}}}\right)$ calculated for $\mathbf{1}-\mathbf{3}$ at different levels of theory.

\begin{tabular}{lllll}
\hline & $J_{\text {dir, }} \mathrm{cm}^{-1}$ & & $J_{1}, \mathrm{~cm}^{-1}$ & $J_{2, \mathrm{~cm}^{-1}}$ \\
& BS-DFT & NEVPT2 & BS-DFT & \\
\hline $\mathbf{1}^{[\mathrm{a}]}$ & -86.1 & $-41.4^{[\mathrm{b}]}$ & & - \\
& -119.0 & $-54.4^{[\mathrm{b}]}$ & - & -0.8 \\
$\mathbf{2}$ & 119.6 & $50.1^{[\mathrm{c}]}$ & 3.7 & -1.6 \\
$\mathbf{3}$ & 47.6 & $18.4^{[\mathrm{c}]}$ & $11.7\left(J_{1}\right), 8.0\left(J_{1}{ }^{\prime}\right)$ & \\
\hline
\end{tabular}

[a] two molecules in the unit cell $[\mathrm{b}](10,10)$ active space; [c] 18 electrons in $15 \mathrm{MO}(18,15)$ active space;

Recently, for the first time for diradicals, the formation of a one-dimensional (1D) antiferromagnetic $S=1$ chain was reported. ${ }^{[2 \mathrm{a}]}$ In turn, diradicals $\mathbf{2}$ and $\mathbf{3}$ are the first examples of a 1D ferromagnetic $S=1$ chain. 


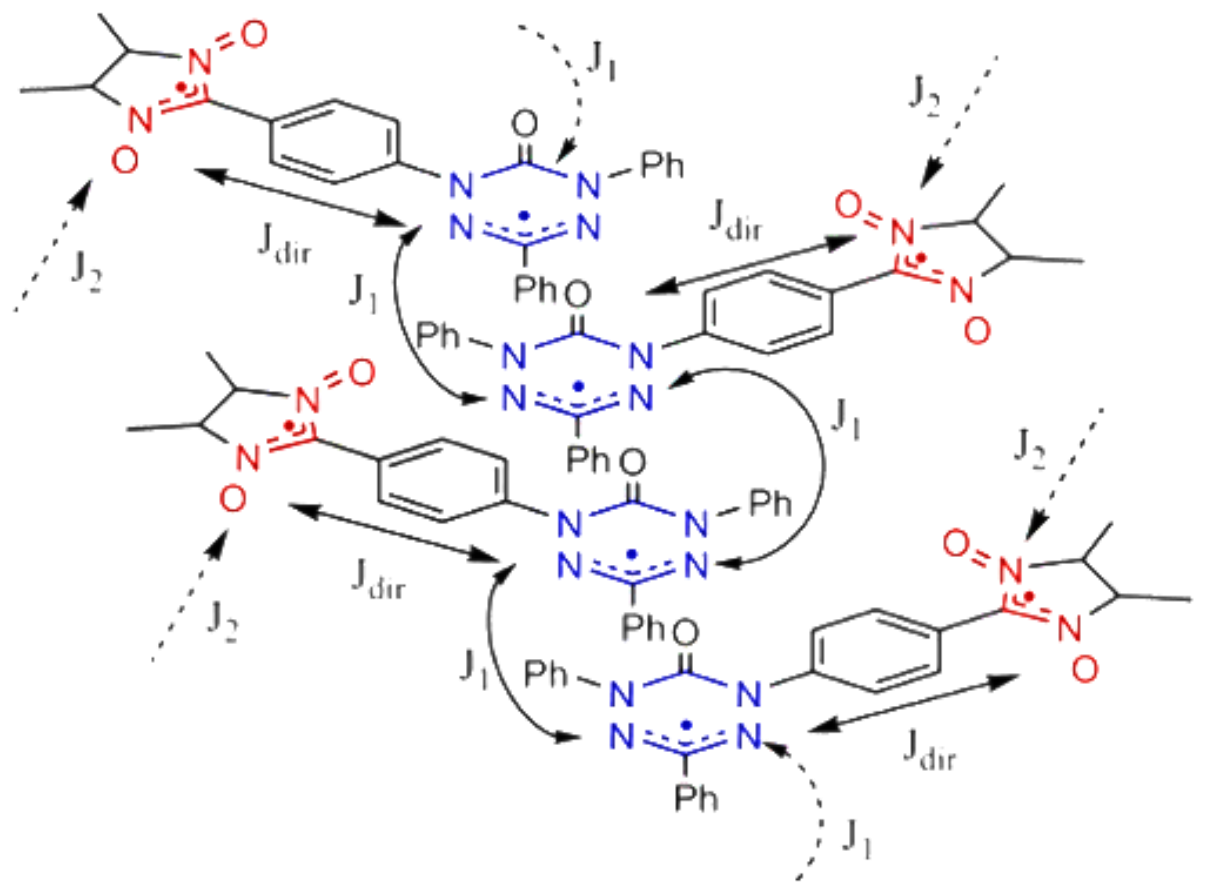

Figure 7. Channels of exchange interactions in the chain of diradicals 2.
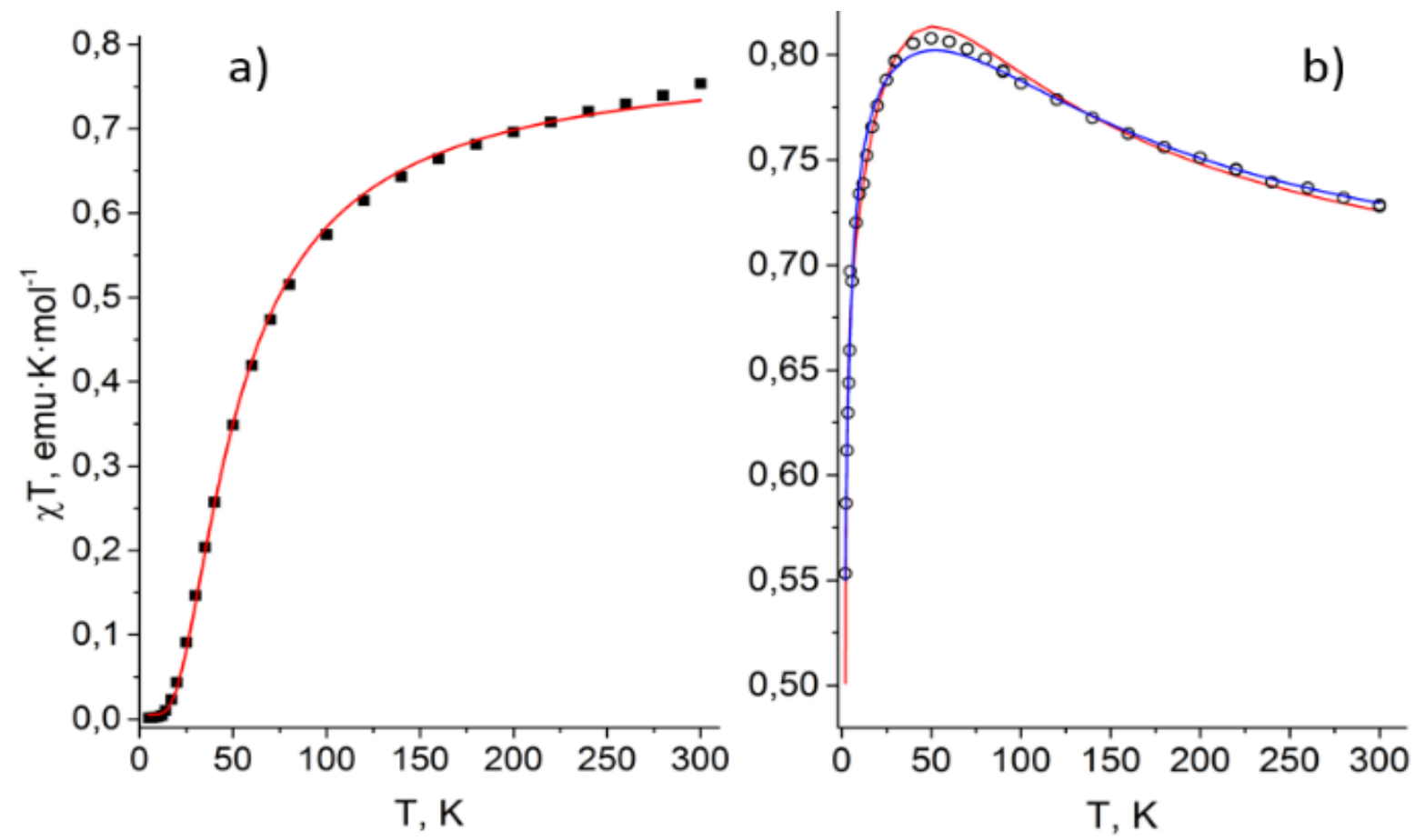

Figure 8. Superconducting quantum interference device (SQUID) magnetometry (circles) of diradicals 1 (a) and 2 (b). The solid curves represent the best theoretical fits (Bleany-Bowers formula: red curves, chain model: blue curve) with the parameters presented in Table 3.

Bulk magnetic properties of mixed verdazyl-nitronyl nitroxide diradicals $\mathbf{1}-\mathbf{3}$ were studied experimentally via SQUID magnetometry of polycrystalline powder samples (Figure 8 and SI Section S5). In all cases, the values of $\mu_{\mathrm{eff}}(T) \approx \sqrt{8 \chi T}$ at $300 \mathrm{~K}$ are close to the theoretical value of $2.45 \mu_{\mathrm{B}}$ for two noninteracting paramagnetic centers with spins $S=1 / 2$ and $g_{\text {iso }}=2$. For diradical 
1, a significant decrease in $\chi \mathrm{T}$ is observed with decreasing temperature, indicating the presence of antiferromagnetic exchange interactions between the paramagnetic centers, in agreement with the calculations (Table 2). For $\mathbf{2}$ and $\mathbf{3}$, a decrease in $\chi \mathrm{T}$ is observed both with a decrease in temperature below and with an increase above $\sim 50 \mathrm{~K}$ (Figure 8 and SI Section S5). This means the presence of moderate ferromagnetic and weak antiferromagnetic interactions. To model the $\chi \mathrm{T}$ temperature dependences, two approaches (isolated diradicals and diradicals aligned in chains) were used, and the application of both leads to good agreement with experiment (Figure 8). The best-fit parameters are summarized in Table 3. A comparison of the data in Table 3 with the calculation results (Table 2) shows that both BS-DFT and high-level approaches correctly predict the sign of exchange interactions in the diradicals. Nonetheless, DFT significantly overestimates the absolute values of $J_{\mathrm{dir}}$, whereas the results of high-level calculations are in perfect agreement with experiment.

Table 3. Parameters of the spin-Hamiltonian for the best theoretical fits of $\chi \mathrm{T}$ temperature dependences of polycrystalline samples of $\mathbf{1}-\mathbf{3}$.

\begin{tabular}{llll|llll}
\hline \multicolumn{3}{l}{ Bleany-Bowers formula with $\theta$} & & \multicolumn{4}{l}{ Chain model with $\theta$} \\
\hline & $J_{\mathrm{dir},} \mathrm{cm}^{-1}$ & $\theta, \mathrm{K}$ & $\mathrm{g}_{\text {iso }}$ & $J_{\mathrm{dir},} \mathrm{cm}^{-1}$ & $J_{1}, \mathrm{~cm}^{-1}$ & $\theta, \mathrm{K}$ & $\mathrm{g}_{\text {iso }}$ \\
\hline $\mathbf{1}$ & $-31.7 \pm 0.3$ & - & 2.070 & - & - & - & - \\
$\mathbf{2}$ & $98 \pm 4$ & $1.01 \pm 0.02$ & 1.806 & 50.1 & 5.5 & -1.5 & 1.886 \\
$\mathbf{3}$ & $19 \pm 4$ & $-5.9 \pm 0.3$ & 2.00 & 12.4 & 3.4 & -5.9 & 1.969 \\
\hline
\end{tabular}

In summary, we synthesized oxoverdazyl-nitronyl nitroxide diradicals 1-3 and revealed their inherent magnetostructural correlations. It was found that the use of the same $p$-phenylene linker gives diradicals $\mathbf{1}$ and $\mathbf{2}$ with singlet or triplet ground states depending on the position of nitroxide moiety in verdazyl ring. Triplet ground state diradical $\mathbf{2}$ has a moderate singlet-triplet energy gap, $\Delta E_{\mathrm{ST}} \approx 0.3 \mathrm{kcal} / \mathrm{mol}$, and high thermal stability with the onset of decomposition at $\sim 190{ }^{\circ} \mathrm{C}$ in an inert atmosphere. $m$-Phenylene-linked triplet diradical $\mathbf{3}$ has a lower singlet-triplet energy gap, $\Delta E_{\mathrm{ST}} \approx 0.07 \mathrm{kcal} / \mathrm{mol}$. Polycrystalline triplet diradicals 2 and $\mathbf{3}$ form a novel type of magnetic packing motif, namely, 1D ferromagnetic $S=1$ chains, and are excellent systems for studying lowdimensional magnetism. The synthesized stable diradicals are important both for the development of verdazyl chemistry and for the creation of new magnetic and spintronic materials based on this type of diradicals. This research opens ways toward high-spin $S=3 / 2$ and $S=2$ organic polyradicals. 


\section{Acknowledgements}

This research was supported by Ministry of Science and Higher Education of the Russian Federation (State Project "Science” №FSWW-2020-0011) and the Russian Science Foundation (grant No. 18-13-00173). D.E.G. and N.P.G. acknowledge support of the Ministry of Higher Education and Science of the Russian Federation (project 0304-2017-0008) and the Irkutsk Supercomputer Centre of the Siberian Branch of the Russian Academy of Sciences for computational resources. Authors would like to acknowledge the Multi-Access Chemical Research Center SB RAS for spectral and analytical measurements. A.S.B. and V.I.O. are thankful to the RSF (grant No. 18-13-00380, magnetochemistry).

Keywords: verdazyl radicals $\bullet$ nitroxides $\bullet$ triplet diradicals $\bullet$ high-spin molecules $\bullet$ ferromagnetic chain

[1] a) M. Baumgarten, High Spin Organic Molecules, in: World Scientific Reference on Spin in Organics, Vol IV, Chap 1 (Ed.: J. S Miller), World Scientific Publishing Co. Pte. Ltd., Singapore, 2018, pp. 1-93; b) S. Suzuki, K. Okada in Organic Redox Systems Synthesis Properties, and Applications (Ed.: T. Nishinaga), Wiley, Hoboken, NJ, 2016, pp. 269-285; c) M. Abe, Chem. Rev. 2013, 113, 7011-7088; d) M. T. Lemaire, Pure and Appl. Chem., 2011, 83, 141149; e) K. Inoue, Metal-Aminoxyl-Based Molecular Magnets, in: $\pi$-Electron Magnetism, From Molecules to Magnetic Materials. Vol. 100 (Ed.: J. Veciana), Springer Berlin Heidelberg, Berlin, Heidelberg, 2001, pp. 61-91; f) I. Fidan, E. Önal, Yu. Yerli, D. Luneau, V. Ahsen, C. Hirel, ChemPlusChem, 2017, 82, 1384-1389; g) R. G. Hicks, Stable Radicals: Fundamentals and Applied Aspects of Odd-Electron Compounds, 2010; h) E. V Tretyakov, V. I. Ovcharenko, Russ. Chem. Rev. 2009, 78, 971-1012; i) S. Kumar, Y. Kumar, S. Keshri, P. Mukhopadhyay, Magnetochemistry 2016, 2, 42; j) T. Akasaka, A. Osuka, S. Fukuzumi, H. Kandori, Y. Aso, Chemical Science of $\pi$-Electron Systems, Springer Japan, Tokyo, 2015; k) T. Nishinaga, Organic Redox Systems: Synthesis, Properties, and Applications, John Wiley \& Sons, Inc, Hoboken, NJ, 2015; 1) E. Coronado, A. J. Epstein, J. Mater. Chem. 2009, 19, 1670-1671.

[2] a) N. Gallagher, H. Zhang, T. Junghoefer, E. Giangrisostomi, R. Ovsyannikov, M. Pink, S. Rajca, M. B. Casu, A. Rajca, J. Am. Chem. Soc. 2019, 141, 4764-4774; b) W. Wang, C. Chen, C. Shu, S. Rajca, X. Wang, A. Rajca, J. Am. Chem. Soc. 2018, 140, 7820-7826; c) N. M. Gallagher, J. J. Bauer, M. Pink, S. Rajca, A. Rajca, J. Am. Chem. Soc. 2016, 138, 9377-9380; d) A. Rajca, A. Olankitwanit, S. Rajca, J. Am. Chem. Soc. 2011, 133, 4750-4753; e) S. Suzuki, T. Furui, M. Kuratsu, M. Kozaki, D. Shiomi, K. Sato, T. Takui, K. Okada, J. Am. Chem. Soc. 2010, 132, 1590815910; f) E. V. Tretyakov, S. E. Tolstikov, G. V. Romanenko, A. S. Bogomyakov, D. V. Stass, A. G. Maryasov, N. P. Gritsan, V. I. Ovcharenko, Russ. Chem. Bull. 2011, 60, 2608-2612. 
[3] W. T. Borden, E. R. Davidson, J. Am. Chem. Soc. 1977, 99, 4587-4594.

[4] Y. Tsuji, R. Hoffmann, M. Strange, G. C. Solomon, Proc. Natl. Acad. Sci. U.S.A. 2016, 113, E413-E419.

[5] a) K. Sugisaki, S. Nakazawa, K. Toyota, K. Sato, D. Shiomi, T. Takui, ACS Cent. Sci. 2019, 5, 167-175; b) S. Nakazawa, S. Nishida, T. Ise, T. Yoshino, N. Mori, R. D. Rahimi, K. Sato, Y. Morita, K. Toyota, D. Shiomi, et al., Angew. Chemie - Int. Ed. 2012, 51, 9860-9864.

[6] D. E. Votkina, P. V. Petunin, S. I. Zhivetyeva, I. Y. Bagryanskaya, M. N. Uvarov, M. S. Kazantsev, M. E. Trusova, E. V. Tretyakov, P. S. Postnikov, European J. Org. Chem. 2020, 2020, 1996-2004.

[7] R. Tanimoto, S. Suzuki, M. Kozaki, K. Okada, Chem. Lett. 2014, 43, 678-680.

Experimental procedures, theoretical calculations, ESR, UV-vis, FT-IR spectra, XRD, SQUID and stability data are included in the supporting information. 